\title{
Concomitant phyllodes tumour and hamartoma of the breast
}

\begin{abstract}
Phyllodes tumours are fibro-epithelial lesions that comprise less than $1 \%$ of breast malignancies. Hamartomas are tumours formed by a cumuli of various epithelial elements, accounting for less than $5 \%$ of benign breast masses. ${ }^{1}$ We present a case of a 47year-old women, with a long standing palpable mass of the left breast whom imaging studies showed two different tumours, with core biopsy diagnosing a phyllodes tumour and fibrocystic changes. The patient underwent partial mastectomy and definitive biopsy confirmed a malignant phyllodes tumour and demonstrated a hamartoma on the other mass. Following surgery, radiotherapy was given. No residual breast lesions have been found in follow-up. To our knowledge, there have not been reported cases of concomitant phyllodes tumour and hamartoma on a single breast.
\end{abstract}

Keywords: phyllodes tumour, hamartoma of the breast, breast MRI
Volume 3 Issue 3 - 2017

\author{
Vial MC,' Maiz C, ${ }^{2}$ Jarry C, ${ }^{4}$ Oddó D, ${ }^{3}$ \\ Camus $\mathrm{M}^{2}$ \\ 'Department of Radiology, Pontificia Universidad Católica de \\ Chile, Chile \\ ${ }^{2}$ Department of Surgical Oncology, Pontificia Universidad \\ Católica de Chile, Chile \\ ${ }^{3}$ Department of Anatomical Pathology, Pontificia Universidad \\ Católica de Chile, Chile \\ ${ }^{4}$ Medicine Student, Pontificia Universidad Católica de Chile, \\ Chile
}

\begin{abstract}
Correspondence: Mauricio Camus A, Department of Surgical Oncology, Pontificia Universidad Católica de Chile, Marcoleta 367, Santiago, Chile, Tel (562) 23543720,
\end{abstract}

Email mcamus@med.puc.cl

Received: June 01, 2017 | Published: July 07, 2017
Abbreviations: PT, phyllodes tumour; LIQ, lower inner quadrant; LOQ, lower outer quadrant; UOQ, union of outer quadrants; MRI, magnetic resonance imaging; EMT, epithelial-mesenchymal transition

\section{Introduction}

Phyllodes tumours (PT) of the breast are rare fibro-epithelial lesions that comprise less than $1 \%$ of all breast malignancies. Based on the degree of stromal hyperplasia and atypia, the World Health Organization (WHO) categorizes PT as benign, borderline and malignant. PT are treated by surgical excision. However, there is no consensus on the definition of an appropriate surgical margin to ensure completeness of excision and reduction of recurrence risk. ${ }^{2}$

Hamartoma is a well circumscribed breast lesion with varying amounts of benign epithelial elements, fibrous tissue, and fat. A simple surgical excision and removal of the entire Hamartoma of breast is normally sufficient treatment for big and symptomatic tumours, but a 'wait and watch' approach may be considered for asymptomatic hamartomas. ${ }^{3,4}$

\section{Case presentation}

A 47year-old woman with history of a long standing palpable mass on the left breast, which was biopsied on 2006 resulting in hamartoma. She had first degree familiar history of breast cancer with her mother diagnosed younger than 50 yyears of age and who later on died of that cause. The patient presented to our office, in June 2016 for progressive growth of her breast tumour. At examination her breasts were fibrous, with a palpable firm and mobile breast mass, not fixed to skin, of $8 \times 6 \times 5 \mathrm{~cm}$ on the left LIQ, and another large, soft and well defined mass in left LOQ and UOQ of $10 \times 10 \times 5 \mathrm{~cm}$, There were no palpable masses in the axilla. On mammography there was a radiolucid well defined breast mass in the left LOQ and UOQ, which was stable in size and shape regarding prior mammogram of twoyears. A new dense, oval and partially defined breast mass was evident in the LIQ (Figure 1). The latter on breast ultrasound corresponded with a hypoechoic and heterogenous nodule with partially defined margins, which measured approximately $7 \mathrm{~cm}$. The first lesion was heterogenous and hyperechoic, measured more than $8 \mathrm{~cm}$, and was the previously biopsied nodule (Figure 2). She later on underwent a breast MRI that showed a round mass in the LIQ, which was hyperintense in T2, hypointense in T1, had a homogenous enhancement with a plateau curve. In the LOQ there was a well-defined mass, which was heterogenous with fibrous and fatty tissue, and did not enhance with gadolinium (Figure 3). A core biopsy of the two masses was then performed, demonstrating a biphasic fibro-epithelial lesion compatible with a PT at the LIQ, and fibrocystic changes and intraductal hyperplasia without atypia at the LOQ. On August 2016, the patient underwent a partial mastectomy, without incidents, and was sent home on the subsequent day (Figure 4). The definitive biopsy demonstrated that the tumour at the LIQ corresponded to a malignant PT (Figures 5) (Figure 6), and that the LOQ mass was a large breast hamartoma (Figures 7 \& Figure 8). Posteriorly, she received 25 radiotherapy fractions, without major adverse effects. She was recently controlled with breast ultrasound and MRI that showed no residual breast lesions. 


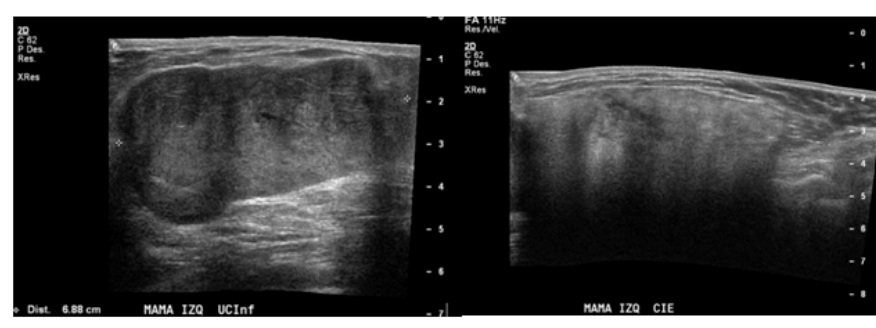

Figure I Preoperative breast ultrasound. A) ULQ of left breast. B) LOQ of the left breast.
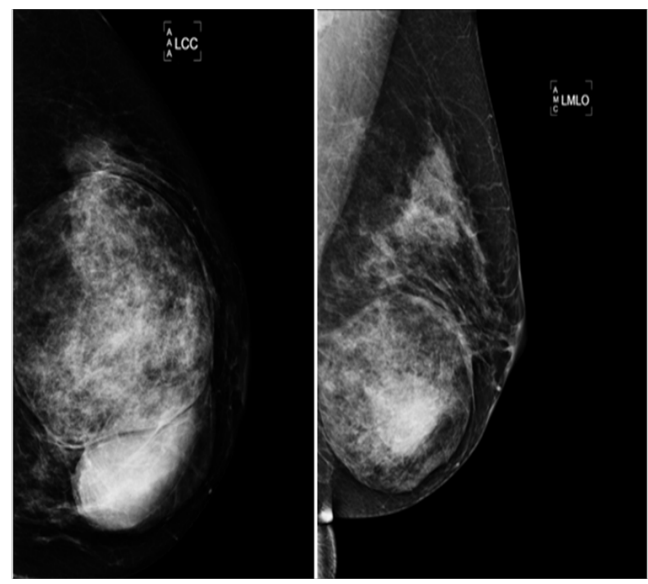

Figure 2 Preoperative mammography. A) Craniocaudal view B) Mediolateral oblique view.

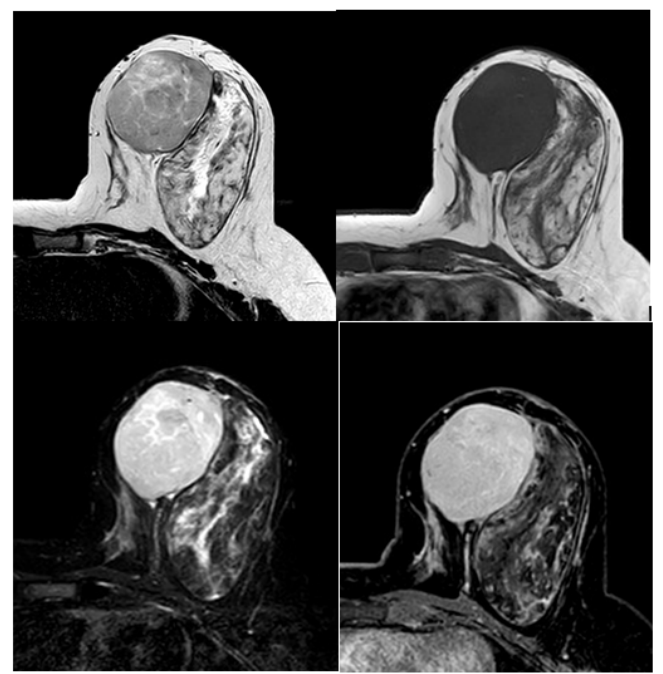

Figure 3 Preoperative breast MRI (T2 weighted image, TI weighted image, STIR and gadolinium enhanced high resolution TIWI with fat saturation).

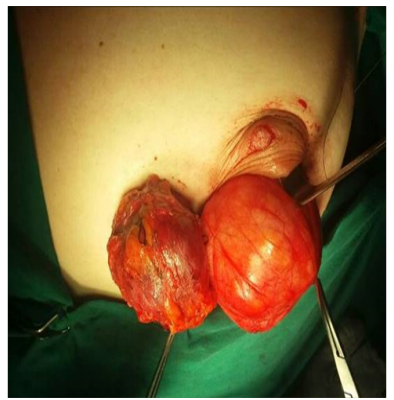

Figure 4 Partial mastectomy, circumareolar incision, showing the excision of both tumours (left Phyllodes and right Hamartoma).

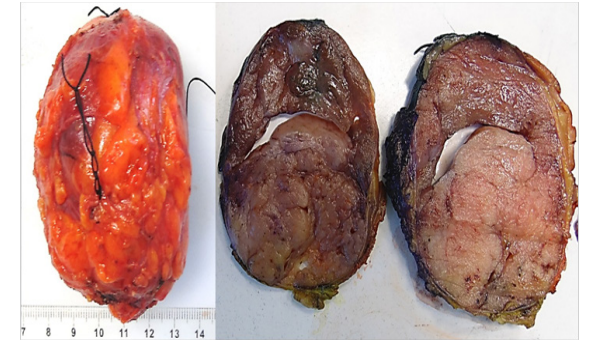

Figure 5 Phyllodes macroscopy. Nodular ovoid mass of $5,2 \times 7,6 \times 6,8 \mathrm{~cm}$ in size and I I 4,2g. To the cut, well delimited, with leaf like protrusions. A) Gross appearance of external surface. B) Gross appearance of cut surface.

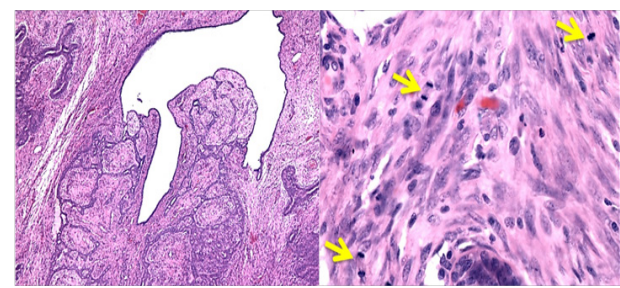

Figure 6 Phyllodes tumour histology with signs of malignancy.A) Microscopic typical appearance. B) The arrows show several mitosis in a microscopic field (haematoxylin - eosin X100, original magnification).

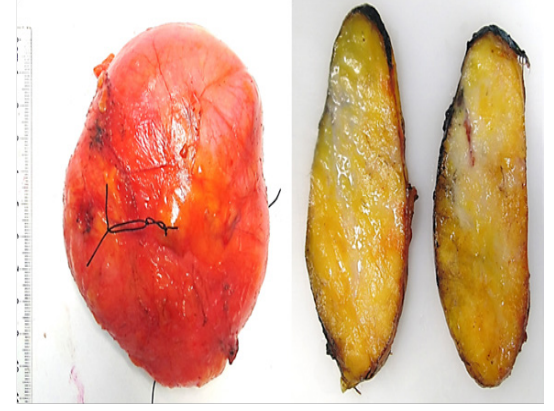

Figure 7 Hamartoma of the breast macroscopy. Nodular ovoid mass of $\mathrm{I} 0, \mathrm{I} \times$ $7,2 \times 4,8 \mathrm{~cm}$ in size and 195.6g. At cut, well delimited, apparently encapsulated and with fibro-adipose aspect. A) Gross appearance of external surface. B) Gross appearance of cut surface.

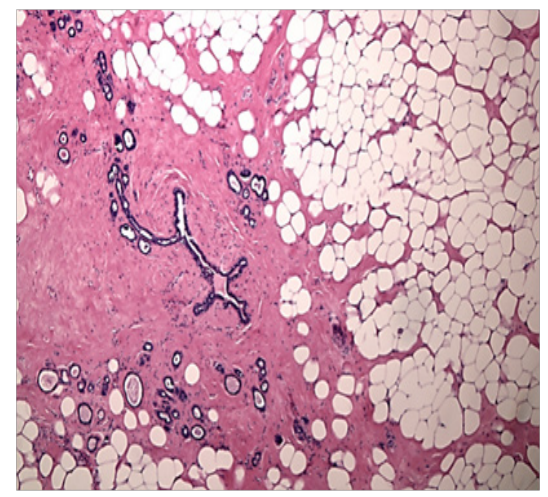

Figure 8 Hamartoma of the breast histology. Microscopic typical appearance (haematoxylin - eosin XI00, original magnification).

\section{Discussion}

The following therapeutic alternatives are proposed for the PT: partial mastectomy with negative margins or total mastectomy. The addition of radiotherapy is under discussion when the probability of local recurrence is high. Partial mastectomy plus radiation therapy was chosen for this patient, instead of total mastectomy, for being 
also curative, based on the slow growing behaviour expected for the phyllodes and considering the patient's lifetime situation. The lesion was resected with a surgical margin between less than $1 \mathrm{~mm}$ to $8 \mathrm{~mm}$ and a very good cosmetic result was obtained. Considering the size of the breast and the size of the tumour, bigger margins would have implied an almost total mastectomy. Although, the risk of relapse was expected to be moderate, radiation therapy was selected for this malignant PT.

Although all degrees of PT have shown local recurrences, the high grade and borderline types clearly have metastatic potential. The prediction of the biological behavior of PT is currently based on their histological type and in particular on the number of mitosis of their stromal (mesenchymal) component, expressed in the amount of mitosis per 10 microscopic fields of major increase, according to classification of the World Health Organization.

Recently, however, the detection of some biomarkers in PT tissue samples, especially markers of epithelial-mesenchymal transition (EMT), was introduced for prognostic and predictive purposes, and in this way to establish therapies complementary to surgery. The studies have considered inducers, regulators and effectors of the EMT process, detected with immunohistochemistry and immunofluorescence techniques. The most important biomarkers have been the snail, slug and twist transcription factors, in addition to the transforming growth factor beta (TGF beta) and cadherins. Studies have shown that the expression of some EMT biomarkers increases correspondingly with the increase in the degree of PT, which may constitute a prognostic value factor; on the other hand, knowledge of the signaling and expression pathways of EMT and the mesenchimal stemness of
PT may represent the target for new complementary therapies of malignant and borderline PT.

\section{Acknowledgements}

None.

\section{Conflict of interest}

Author declares that there is no conflict of interest.

\section{References}

1. Charpin C, Mathoulin MP, Andrac L, et al. Reappraisal of breast hamartomas. A morphological study of 41 cases. Pathol Res Pract. 1994;190(4):362-371

2. Tan BY, Acs G, Apple SK, et al. Phyllodes tumours of the breast: a consensus review. Histopathology. 2016;68(1):5-21.

3. Tse GM, Law BKB, Ma TKF, et al. Hamartoma of the breast: a clinicopathological review. J Clin Pathol. 2002;55(12):951-954.

4. Tatar C, Erözgen E, Tüzün S, et al. Surgical Approach to Breast Hamartoma and the Diagnostic Accuracy in Preoperative Biopsies. J Breast Health. 2013;9:186-190.

5. Fen X, Zhao L, Shen H, et al. Expression of EMT markers and mode of surgery are prognostic in phyllodes tumors of the breast. Oncotarget. 2017;8(20):33365-33374.

6. Kwon JE, Jung WK, Koo JS. Molecules involved in epithelial-mesenchymal transition and epithelial-stromal interaction in phyllodes tumors: implications for histologic grade and prognosis. Tumor Biol. 2012;33(3):787-798 